\title{
Dichotomy in intrahepatic cholangiocarcinomas based on histologic similarities to hilar cholangiocarcinomas
}

\author{
Masayuki Akita ${ }^{1,2}$, Kohei Fujikura ${ }^{1}$, Tetsuo Ajiki ${ }^{2}$, Takumi Fukumoto ${ }^{2}$, Kyoko Otani ${ }^{1}$, \\ Takeshi Azuma ${ }^{3}$, Tomoo Itoh ${ }^{1}$, Yonson $\mathrm{Ku}^{2}$ and Yoh Zen ${ }^{1}$ \\ ${ }^{1}$ Department of Diagnostic Pathology, Kobe University Graduate School of Medicine, Kobe, Japan; \\ ${ }^{2}$ Department of Hepato-Biliary-Pancreatic Surgery, Kobe University Graduate School of Medicine, Kobe, Japan \\ and ${ }^{3}$ Department of Gastroenterology, Kobe University Graduate School of Medicine, Kobe, Japan
}

\begin{abstract}
Intrahepatic cholangiocarcinomas were classified into two types based on their microscopic appearance. Tumors with histologic similarities to hilar cholangiocarcinomas (predominantly ductal adenocarcinomas with minor tubular components, if present, restricted to the invasive front) were defined as the perihilar type, whereas the others were classified as peripheral cholangiocarcinomas. Among the 47 cases examined in the present study, $26(55 \%)$ were classified as the perihilar type, whereas $21(45 \%)$ were the peripheral type. The perihilar type had higher pT stages and more frequently showed a periductal-infiltrating gross appearance and microscopic perineural infiltration than peripheral cholangiocarcinomas. The presence of low-grade biliary intraepithelial neoplasia in the adjacent bile ducts was only found in perihilar cholangiocarcinomas $(6 / 21,29 \%)$. The immunophenotype also differed between the two types with MUC5AC and MUC6 being more commonly expressed in the perihilar type. One-third of perihilar cholangiocarcinomas lacked the expression of SMAD4, suggesting SMAD4 mutations, whereas the loss of BAP1 expression and IDH1 mutations were almost restricted to the peripheral type ( 35 and $15 \%$, respectively). Patients with perihilar cholangiocarcinoma had worse overall survival than those with peripheral cancer $(P=0.027)$. A multivariate analysis identified the histologic classification as an independent prognostic factor $(P=0.005$, $H R=3.638)$. Comparisons between intrahepatic and hilar cholangiocarcinomas also revealed that the molecular features and prognosis of perihilar cholangiocarcinomas were very similar to those of hilar cholangiocarcinomas. In conclusion, this histologybased classification scheme of intrahepatic cholangiocarcinomas will be useful and clinically relevant because it represents different underlying molecular features and has an independent prognostic value.

Modern Pathology (2017) 30, 986-997; doi:10.1038/modpathol.2017.22; published online 24 March 2017
\end{abstract}

Intrahepatic cholangiocarcinoma is the second most common primary liver cancer and has a significantly worse prognosis than that of hepatocellular carcinoma. ${ }^{1,2}$ Although most intrahepatic cholangiocarcinomas are morphologically adenocarcinomas, their molecular and biological features are likely to be heterogeneous. Recent studies have suggested that there are two subtypes of intrahepatic cholangiocarcinomas with distinct clinicopathologic features. ${ }^{3-5}$ One type suspected of originating from small bile ducts or ductules frequently develops against the

Correspondence: Professor Y Zen, MD, PhD, FRCPath, Department of Diagnostic Pathology, Kobe University Graduate School of Medicine, 7-5-2 Kusunoki-cho, Kobe 650-0017, Japan.

E-mail yohzen@med.kobe-u.ac.jp

Received 10 December 2016; revised 23 February 2017; accepted 24 February 2017; published online 24 March 2017 background of chronic liver diseases such as viral hepatitis or alcoholic cirrhosis. Histologically, tumors in that group mainly consist of tubular adenocarcinomas with scant mucus, somewhat reminiscent of reactive bile ductules. The other type, which has been suggested to be derived from the large bile ducts, is characterized by a more central location with the morphologic appearance of sclerosing adenocarcinoma with tumor cells arranged in large ducts. Some of the latter cases have biliary intraepithelial neoplasia in the adjacent ducts, suggesting multi-step carcinogenesis. Several terms have been proposed to describe these two types. ${ }^{3-5}$ In the present study, the former is hereafter referred to as peripheral cholangiocarcinomas and the latter as perihilar cholangiocarcinomas.

Although similar pathologic differences between the two groups have been identified in previous 
studies, the separation criteria used have varied among studies. Cases have been classified based on the anatomical location, ${ }^{3,6}$ histologic appearance (tubular or ductal), ${ }^{4}$ or expression patterns of immunohistochemical markersm, such as S100P, $\mathrm{N}$-cadherin, and tubulin $\beta$-III. ${ }^{5,7-9}$ However, none of these criteria are universally accepted. In clinical settings, distinctly nodular tumors in the peripheral liver parenchyma without recognizable large-duct involvement may be regarded as peripheral cholangiocarcinomas. Similarly, periductal-infiltrating tumors mostly confined to Glisson's capsule of large bile ducts are most likely to be perihilar cholangiocarcinomas. However, a large number of cases involve both the liver parenchyma and periductal connective tissue. Therefore, it remains challenging to accurately classify these tumors on a case-bycase basis.

In the present study, we attempted to separate intrahepatic cholangiocarcinomas into two types based on a morphologic standard. According to our hypothesis that perihilar cholangiocarcinomas may be biologically similar to hilar cholangiocarcinomas, we initially reviewed the histology of hilar cholangiocarcinomas to elucidate their morphologic features. The obtained findings were applied to the classification criteria, in which perihilar intrahepatic cholangiocarcinomas were defined as intrahepatic neoplasms that are morphologically similar to hilar cholangiocarcinomas. The clinicopathologic and molecular features of three types of cholangiocarcinomas (peripheral, perihilar, and hilar) were then compared to confirm whether our classification scheme is clinically relevant.

\section{Materials and methods}

\section{Case Selection}

This study was approved by the Ethics Committee at Kobe University Graduate School of Medicine (\#160090). The study cohort consisted of 47 consecutive patients with intrahepatic cholangiocarcinoma who underwent liver resection at Kobe University Hospital between 2000 and 2015, and 32 patients with hilar cholangiocarcinoma who underwent surgical resection between 2008 and 2014 . Cases showing a cholangiolocellular pattern with no hepatocellular components were included as intrahepatic cholangiocarcinomas in the present study, while classical combined hepatocellularcholangiocarcinomas were excluded. Intraductal papillary neoplasms of the bile ducts were also excluded because they have distinct features from conventional intrahepatic cholangiocarcinomas. ${ }^{10}$ Hilar cholangiocarcinoma was defined as a tumor predominantly involving the right and left hepatic ducts and their junction, whereas tumors more proximal in location were called intrahepatic cholangiocarcinomas.

\section{Classification of Intrahepatic Cholangiocarcinomas}

A histology review of hilar cholangiocarcinomas revealed that all tumors mainly consisted of ductal adenocarcinomas, in which columnar, often clear tumor cells were arranged in large ducts with intraductal and/or cytoplasmic mucus. Although tubular components (resembling bile ductules) were also occasionally present, they were always observed at the invasive front, particularly the interface between the tumor and liver parenchyma. This growth pattern was never identified in the central parts of hilar cholangiocarcinomas. On the basis of these results, perihilar cholangiocarcinomas were defined as adenocarcinomas with a predominantly ductal morphology and minor tubular components, if present, restricted to the tumor-liver interface. Intrahepatic cholangiocarcinomas beyond these criteria were called peripheral cholangiocarcinomas.

On the basis of these criteria, two doctors (MA and YZ) read the slides and independently classified each case. Concordant diagnoses were achieved in 44 of 47 cases. For three cases with discrepant interpretations, a consensus opinion was reached for discrepant cases after discussions using a multiheader microscope. The cohort was also reviewed by another pathologist $(\mathrm{KO})$ to evaluate reproducibility of the histological classification. The concordant rate and kappa value of interpretations among the three pathologists were $84 \%$ and 0.688 , respectively.

\section{Evaluation of Clinicopathologic Features}

Electronically stored clinical records were reviewed in terms of a history of chronic liver diseases, serum concentrations of tumor markers, and patient prognoses. Tumors were classified into mass-forming, periductal-infiltrating, and mixed types based on gross pictures. The mass-forming type was defined as distinctly nodular tumors, whereas the periductalinfiltrating type mainly involved Glisson's capsule around intrahepatic large bile ducts. Nodular tumors with extranodular extension along periductal connective tissue were called the 'mixed type'. Other pathologic parameters examined were tumor size, the degree of differentiation, microscopic lymphovascular and perineural invasion, intrahepatic metastasis, and lymph node metastasis. The presence or absence of biliary intraepithelial neoplasia 1 or 2 in the adjacent bile ducts was also evaluated as a histologic sign suggesting multi-step carcinogenesis. Biliary intraepithelial neoplasia 3 was not counted because the discrimination between pre-malignant biliary intraepithelial neoplasia 3 and secondary mucosal infiltration by an invasive cancer (cancerization) is currently impossible based on histologic evidence. Mucicarmine stain was also performed to evaluate mucin production. Each case was staged according to the 7th edition of the UICC/AJCC TNM classification system. ${ }^{11}$ 


\section{Immunohistochemistry}

All samples were fixed in 10\% buffered formalin and embedded in paraffin. Tissue microarrays of formalin-fixed paraffin-embedded tumor tissue were constructed for immunohistochemistry. Three tissue cores ( $2 \mathrm{~mm}$ in diameter) were taken from each case. Immunostaining was performed on a Ventana Benchmark XT (Ventana Medical Systems, Tucson, AZ, USA) or Bond Max autostainer (Leica Microsystems, Wetzlar, Germany) according to the manufacturers' protocols. All cases were stained for MUC1, MUC2, MUC5AC, MUC6, CK7, CK20, p53, BAP1, and SMAD4. MUCs and CKs were selected to characterize tumor cell phenotypes. Immunostaining for SMAD4, BAP1, and p53 was performed to evaluate the mutation status of these genes because immunohistochemical findings of SMAD4, BAP1, and p53 are known to strongly correlate with genetic alterations. $^{12,13}$

Deparaffinized sections were heat-treated and incubated with primary antibodies. The antibodies used were as follows: MUC1 (clone Ma695; dilution 1:100, Leica Microsystems), MUC2 (clone Ccp58; dilution 1:100, Leica Microsystems), MUC5AC (clone CLH2; dilution 1:100, Leica Microsystems), MUC6 (clone CLH5; dilution 1:100, Leica Microsystems), CK7 (clone OV-TL 12/30; dilution 1:100, Dako Cytomation, Glostrup, Denmark), CK20 (clone Ks20.8; dilution 1:50, Dako Cytomation), SMAD4 (clone B-8; dilution 1:100, Santa Cruz Biotechnology, Santa Cruz, CA), BAP1 (clone C-4; dilution 1:100, Santa Cruz Biotechnology), p53 (clone DO-7; dilution 1:300, Leica Microsystems), S100P (clone 357517; dilution 1:500, R\&D systems, Minneapolis, USA), N-cadherin (clone IAR06; dilution 1:100, Leica Microsystems), and NCAM (clone Ccp58; 1:100, Novocastra Laboratories, Newcastle, UK).

Expression levels were evaluated semiquantitatively according to the percentages of positive cells: 0 (negative); $1+$ (focal), $1-5 \% ; 2+$ (moderate), 6-50\%; $3+$ (diffuse), more than 50\%. Cases with $2+$ and $3+$ expression levels were considered to be positive for MUCs and CKs. Regarding p53, the nuclear staining of $3+$ was regarded as a positive result, indicating a p53 mutation. Cases with no detectable cytoplasmic or nuclear SMAD4 protein were scored as negative for SMAD4.12 The completely negative nuclear staining of BAP1 was defined as a loss of expression, suggesting a $B A P 1$ mutation. ${ }^{13}$ S100P, N-cadherin, and NCAM immunohistochemistry were evaluated according to a previous report. ${ }^{5}$

\section{Mutational Analysis of $K R A S$ and $I D H 1 / 2$}

Genomic DNA was extracted from formalin-fixed paraffin-embedded tissue blocks. Areas consisting predominantly of tumor cells were selected for DNA extraction under a microscope. DNA was extracted using the QIAamp DNA FFPE Tissue Kit (Qiagen, Hilden, Germany). DNA content was assessed using a NanoDrop 2000c spectrophotometer (Thermo Fisher Scientific, Waltham, MA, USA).

KRAS (exons 2 and 3 ) and $I D H 1 / 2$ were amplified by PCR using primers designed to produce one amplicon of a mutational hotspot. The primer sequences used were: forward 5'-AGGCCTGCTGA AAATGACTG-3' and reverse 5'-GGTCCTGCACCA GTAATATGCA-3' for exon 2 of $K R A S$; forward $5^{\prime}-\mathrm{C}$ CAGACTGTGTTTCTCCCTTCTC-3' and reverse 5'-AGAAAGCCCTCCCCAGTCCTCA-3' for exon 3 of KRAS; forward 5'-AAACAAATGTGGAAATCAC C-3' and reverse 5'-TGCCAACATGACTTACTTG A-3' for IDH1 codon 132; forward 5'-AGAAGAT GTGGAAAAGTCCC-3' and reverse 5'-CAGAGAC AAGAGGATGGCTAGG-3' for IDH2 codon 172. PCR was performed using 10-40 ng of genomic DNA, 10 pmol each of the forward and reverse primers (Hokkaido System Science, Hokkaido, Japan), and $15 \mu \mathrm{l}$ of GoTaq Green Master Mix $2 \times$ (Promega, Madison, WI, USA) in a total volume of $30 \mu \mathrm{l}$. The PCR thermocycling conditions used were as follows: $95^{\circ} \mathrm{C}$ for $1 \mathrm{~min}$, followed by 35 cycles of $95^{\circ} \mathrm{C}$ for $30 \mathrm{~s}, 56^{\circ} \mathrm{C}$ for $30 \mathrm{~s}$, and $72^{\circ} \mathrm{C}$ for $1 \mathrm{~min}$. Amplification products were purified using the Wizard SV Gel and PCR Clean-up System Kit (Promega). Cycle sequencing was performed using BigDye Terminator v3.1 (Applied Biosystems, Foster City, CA, USA) and the amplified products were separated by capillary electrophoresis on an ABI 3130xl Genetic Analyzer (Applied Biosystems). Sequencing results were analyzed using Sequencing Analysis 5.2 and SeqScape software (Applied Biosystems).

\section{Statistical Analysis}

Continuous variables were assessed by an unpaired $t$-test and $\chi^{2}$ test. Continuous and categorical variables in each group were compared using Fisher's exact test. Overall survival was calculated using the Kaplan-Meier method. Univariate and multivariate analyses were performed on the prognostic factors assessed by Cox's proportional hazards model. JMP 12 (SAS Institute, Cary, NC, USA) was used for the statistical analysis.

\section{Results}

\section{Clinicopathologic Findings}

Among the 47 cases of intrahepatic cholangiocarcinomas examined, $21(45 \%)$ were classified as the perihilar type, whereas 26 (55\%) were the peripheral type, according to the pathologic features shown in Figures 1 and 2. All peripheral cholangiocarcinomas contained foci of tubular adenocarcinomas in central areas of the tumor (Figures $2 \mathrm{~b}$ and $\mathrm{c}$ ), whereas this growth pattern was not present $(n=14,67 \%)$ or only focally observed at the tumor-liver interface $(n=7$, $33 \%$ ) in perihilar cancers by definition (Figure 1d). Four cases $(15 \%)$ of peripheral cholangiocarcinomas 

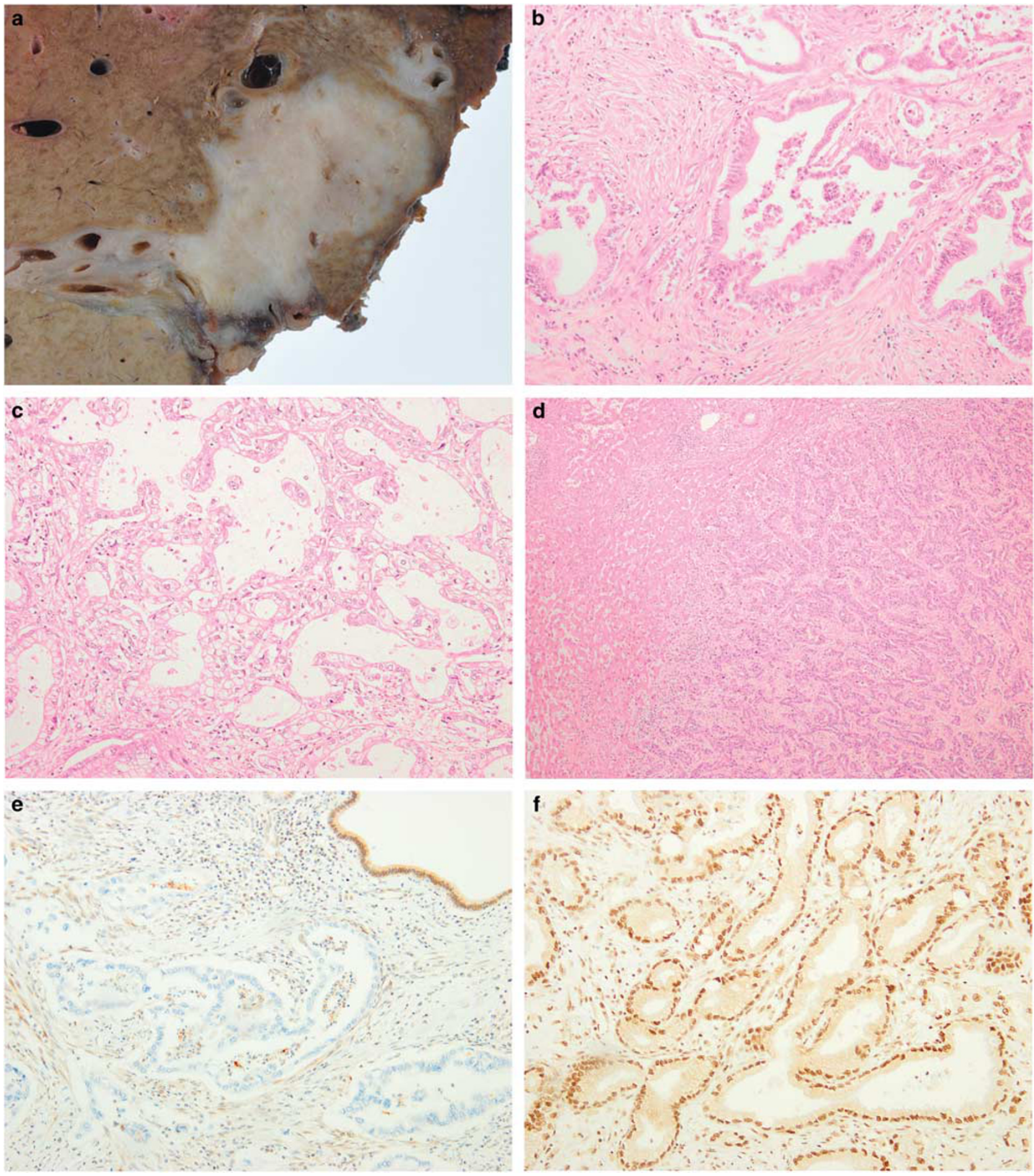

Figure 1 Pathologic features of perihilar cholangiocarcinomas. (a) On cut sections, the nodular tumor focally extends along periductal connective tissue, consistent with the mixed mass-forming and periductal-infiltrating type. (b) The tumor consists of duct-forming adenocarcinoma and abundant fibrotic stroma. (c) Cancer cells have a clear cytoplasm. (d) Tubular components similar to reactive bile ductules are observed at the tumor-liver interface of the invasive front. (e) The expression of SMAD4 is lost in cancer cells, but is preserved in the non-neoplastic epithelium (right upper field). (f) BAP1 is strongly expressed in the cytoplasm and nuclei.

consisted of almost entirely tubular carcinoma with an anastomosing ductular architecture, consistent with a cholangiolocellular-carcinoma pattern. Five cases (two perihilar and three peripheral cholangiocarcinomas) contained foci of poorly differentiated solid adenocarcinomas; however, tubular and/or ductal components were also present elsewhere and histologic types were evaluated on the 

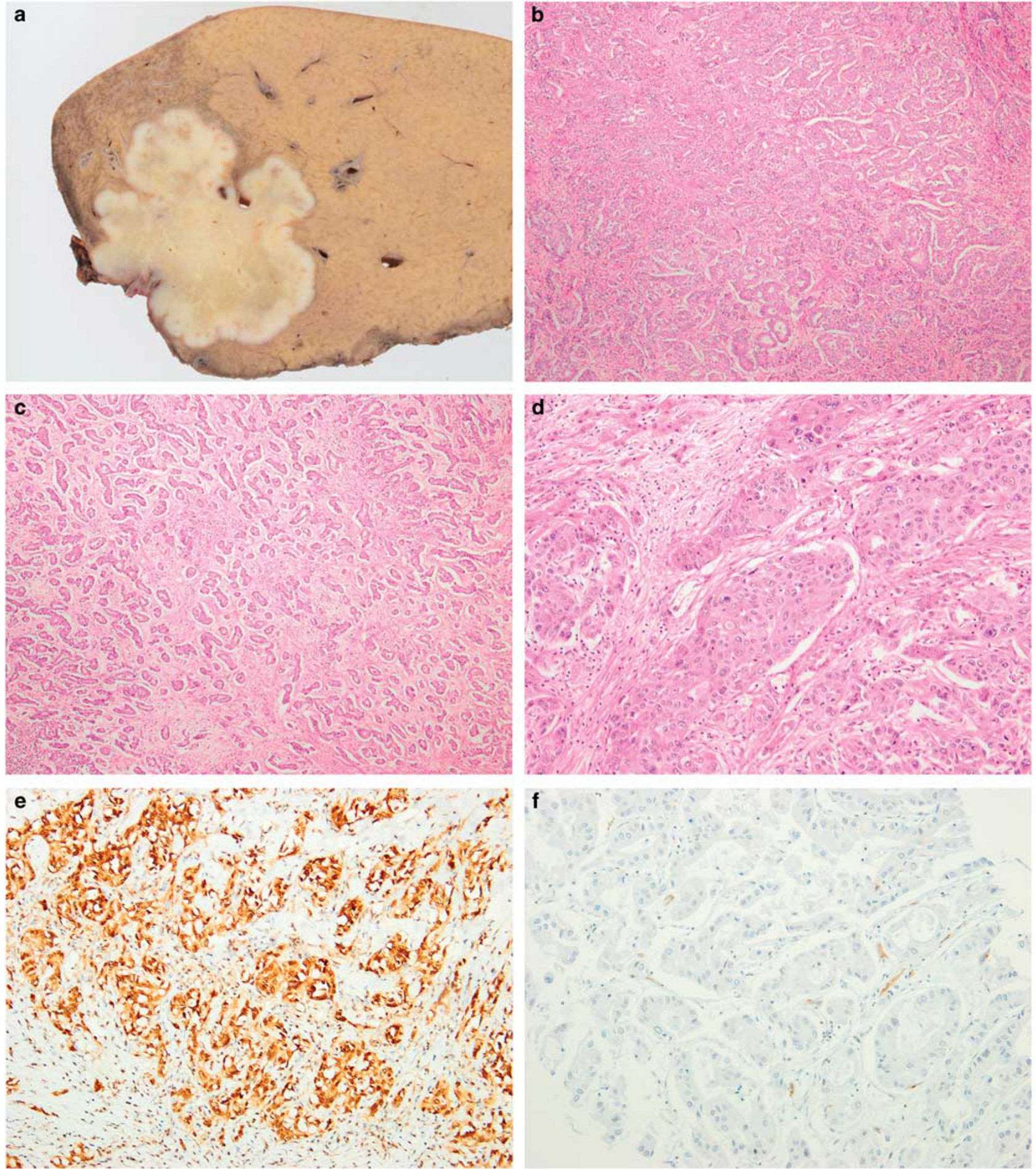

Figure 2 Pathologic features of peripheral cholangiocarcinomas. (a) The lobular tumor is located inside the liver parenchyma (massforming type). (b) Tumor cells are arranged in an irregularly anastomosing tubular architecture with scant mucin. (c) Some parts show a cholangiolocellular pattern. (d) A hepatoid appearance is focally observed. (e) The expression of SMAD4 is well preserved. (f) Cancer cells are entirely negative for BAP1.

more differentiated areas. Although another case had a hepatoid element, in which cancer cells were arranged in a thick-trabecular architecture (Figure 2d), immunohistochemistry for hepatocellular markers (Heppar-1, Arginase-1, and bile salt export pump [BSEP]) was negative. No patients in our cohort had a history of preceding biliary disease, such as primary sclerosing cholangitis, intrahepatic stones, or liver fluke infestation.

Table 1 compares clinicopathologic features between the two types. Patients with perihilar cholangiocarcinoma were more commonly female 
Table 1 Comparisons of clinicopathologic features among peripheral, perihilar, and hilar cholangiocarcinomas

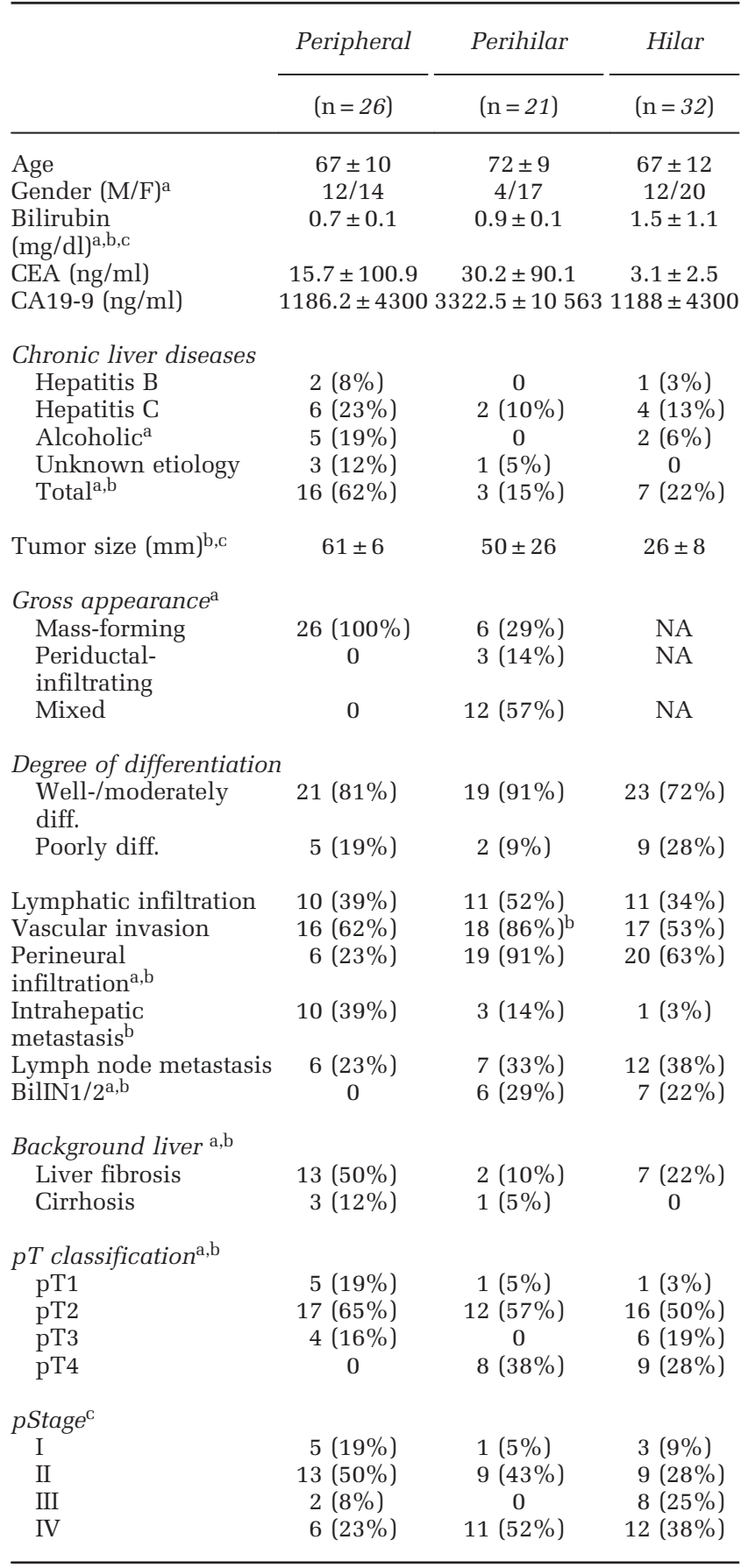

Abbreviations: BilIN, biliary intraepithelial neoplasia; CEA, carcinoembryonic antigen; CA19-9, carbohydrate antigen 19-9; NA, not applicable.

${ }^{a} P<0.05$ peripheral vs perihilar cholangiocarcinomas.

${ }^{\mathrm{b}} P<0.05$ peripheral vs hilar cholangiocarcinomas.

${ }^{\mathrm{c}} P<0.05$ perihilar vs hilar cholangiocarcinomas.

and had higher serum concentrations of bilirubin than those with peripheral cholangiocarcinoma. Peripheral cholangiocarcinomas more frequently correlated with chronic liver diseases than with perihilar cancers (62 vs 15\%, $P=0.003$ ). The background liver of peripheral cholangiocarcinomas showed septal/bridging fibrosis $(n=13,50 \%)$ or cirrhosis $(n=3,12 \%)$.

Grossly, all peripheral cholangiocarcinomas showed mass-forming tumors. Although the periductal extension was observed at least focally in 15 $(71 \%)$ perihilar cholangiocarcinomas, the remaining $6(29 \%)$ were classified as the mass-forming type. Intraductal growth was focally noted in a case of periductal-infiltrating perihilar cancer. Histologically, perihilar cholangiocarcinomas had higher pT stages and more frequent perineural infiltration than peripheral cholangiocarcinomas (both $P<0.001$ ). The presence of biliary intraepithelial neoplasia 1 or 2 in the adjacent bile ducts was only noted in perihilar cholangiocarcinomas $(6 / 21,29 \%)$. In contrast, intrahepatic metastases including daughter nodules were slightly more frequent in peripheral cholangiocarcinomas $(10 / 26,38 \%)$ than in perihilar cancers $(3 / 21,14 \%)(P=0.059)$.

Hilar cholangiocarcinomas were also compared with two types of intrahepatic cholangiocarcinomas. Hilar cancers were more similar to the perihilar type than to the peripheral type, particularly in terms of the association with biliary intraepithelial neoplasia $1 / 2$, frequent perineural infiltration, and the less common association with liver fibrosis.

\section{Immunohistochemistry}

As shown in Figure 3, 16/21 (76\%) perihilar cholangiocarcinomas expressed MUC5AC, whereas only $2 / 26(8 \%)$ peripheral cancers were positive for this mucin $(P<0.001)$. Similarly, MUC6 was more frequently expressed in perihilar cholangiocarcinomas than in peripheral cancers $(6 / 21(29 \%)$ vs $1 / 26$ $(4 \%), P=0.015)$. No significant difference was observed between the two groups regarding the expression of MUC1, MUC2, CK7, and CK20. The immunophenotype of tumor cells in perihilar cholangiocarcinomas appeared to be similar to that in hilar cholangiocarcinomas, with no marked differences being observed in any of the molecules examined (Figure 3).

The loss of SMAD4 expression indicating gene mutations was noted in 7/21 (33\%) perihilar cholangiocarcinomas, whereas it was observed in a single case $(4 \%)$ of peripheral cholangiocarcinoma $(P=0.006$; Figures $1 \mathrm{e}$ and $2 \mathrm{e})$. The results of BAP1 immunohistochemistry were the opposite, with the loss of its expression being highly specific to peripheral cholangiocarcinomas $(9 / 26 \quad(35 \%)$ vS $1 / 21 \quad(5 \%) \quad P=0.008$ ) (Figures $1 \mathrm{f}$ and 2f). The extensive expression of p53 was similarly observed in both types: 7/21 (33\%) perihilar and 7/26 (27\%) peripheral cholangiocarcinomas.

In hilar cholangiocarcinomas, the expression of SMAD4 was lost in 9/32 (28\%) cases, whereas that of BAP1 was retained in all cases. The strong nuclear 
MUC1

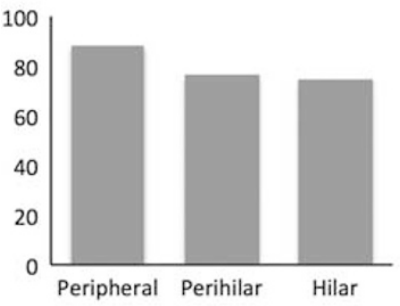

MUC6

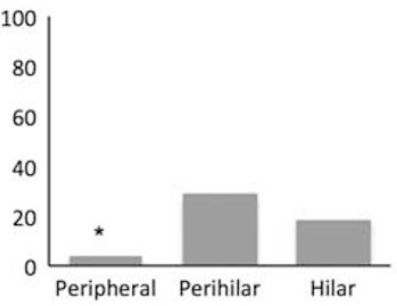

p53

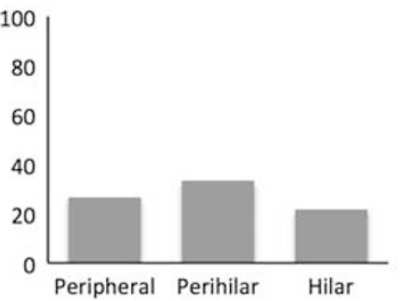

MUC2

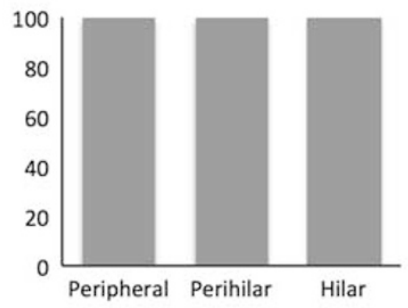

CK7

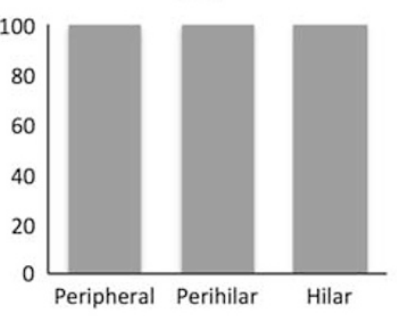

Loss of SMAD4

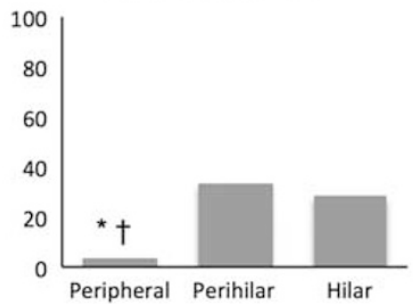

MUC5AC
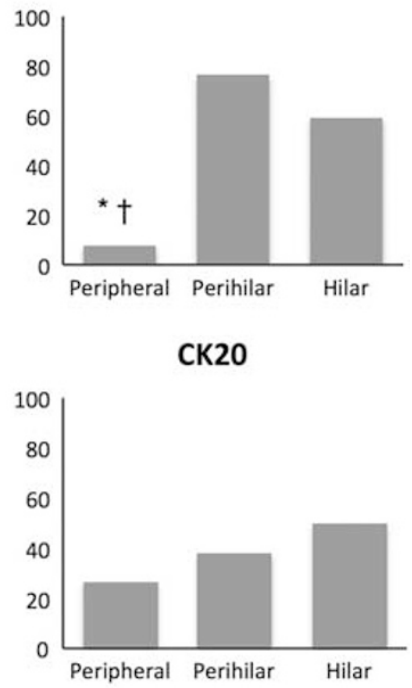

Loss of BAP1

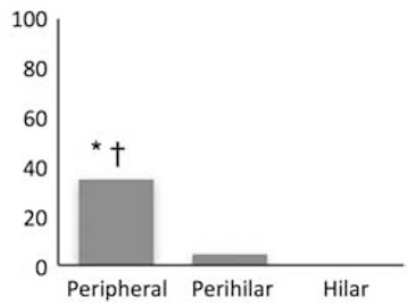

Figure 3 Results of immunohistochemistry. The expression of MUC5AC and MUC6 in perihilar and hilar cholangiocarcinomas was significantly stronger than that in the peripheral type. The loss of SMAD4 expression was more commonly observed in perihilar and hilar cholangiocarcinomas, whereas the expression of BAP1 was more frequently lost in peripheral cholangiocarcinomas. ${ }^{*} P<0.05 v s$ perihilar cholangiocarcinomas; ${ }^{\dagger} P<0.05$ vs hilar cholangiocarcinomas.

expression of p53 was observed in $7 / 32(22 \%)$ cases. These results in hilar cholangiocarcinomas were similar to those in perihilar cancers.

\section{Mutational Analysis of KRAS and IDH1/2}

Exons 2 and 3 of KRAS were sequenced in all cases. The 14 mutations identified were G12D $(n=9), \mathrm{G} 12 \mathrm{~V}$ $(n=2)$, G12R $(n=1)$, G13D $(n=1)$, and Q61H $(n=1)$ (Figure 4). The frequencies of KRAS mutations did not significantly differ between the two types of intrahepatic cholangiocarcinomas (14 vs 19\%; $P=0.652)$. KRAS was also mutated at a similar frequency in hilar cholangiocarcinomas (6/32, 19\%).

IDH1 and IDH2 genes were successfully sequenced in all cases. Codon 132 of IDH1 was mutated in four cases, which were all peripheral cholangiocarcinomas (Figure 4). Therefore, the frequency of IDH1 mutations was significantly higher in peripheral cholangiocarcinomas than in perihilar cancers (15 vs $0 \% ; P=0.025$ ). All mutations identified were R132C. IDH1 was the wild type in all hilar cholangiocarcinomas. KRAS and IDH1 mutations were mutually exclusive with no case harboring both genetic alterations. No mutation in $I D H 2$ was observed in our cohort.

\section{Outcomes After Surgery and Prognostic Factors}

Twenty-three patients with intrahepatic cholangiocarcinoma died during the mean follow-up period of 31 months. The overall survival rates of patients with intrahepatic cholangiocarcinoma were $71 \%$ at 1 year, $53 \%$ at 3 years, and $42 \%$ at 5 years after surgery. Overall survival of patients with perihilar cholangiocarcinoma was significantly worse than those with peripheral cancer $(P=0.027)$. The 5 -year overall survival rate was $21 \%$ for perihilar cholangiocarcinomas and $63 \%$ for peripheral cholangiocarcinomas (Figure 5). Survival curves were similar in the first 2 years, with differences becoming more apparent thereafter. Four patients with IDH1mutated cholangiocarcinoma were alive, with recurrence being observed in a single case at the time of writing. In comparisons with intrahepatic and hilar cholangiocarcinomas, the survival curve of patients with perihilar cholangiocarcinoma appeared to be similar to that of patients with hilar cholangiocarcinoma (Figure 5).

To elucidate whether the histologic classification of intrahepatic cholangiocarcinomas is an independent prognostic factor, a multivariate analysis was performed with the application of four other 
a

\begin{tabular}{llll}
\hline & Peripheral $(n=26)$ & Perihilar $(n=21)$ & Hilar $(n=32)$ \\
\hline KRAS & & & \\
Mutation & $5(19 \%)$ & $3(14 \%)$ & $6(19 \%)$ \\
Amino acid substitution & G12D (3), G12V (1), Q61H (1) & G12D (2), G13D (1) & G12D (4), G12R (1) G12V(1) \\
IDH1 & & & \\
Mutation & $4(15 \%)^{*+}$ & 0 & 0 \\
Amino acid substitution & R132C (4) & NA & NA \\
\hline
\end{tabular}

b

\section{KRAS}

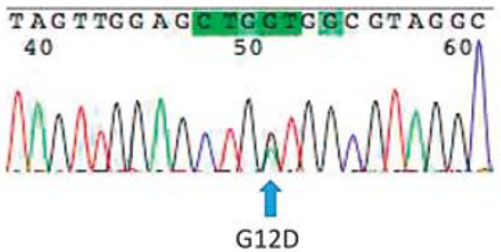

C

IDH1
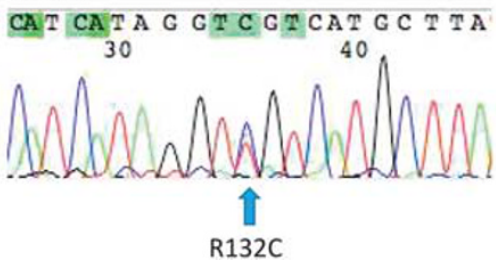

Figure 4 KRAS and IDH1 mutations. (a) The frequencies and resultant amino acid substitutions of gene mutations are summarized. ${ }^{*} P<0.05$ vs perihilar cholangiocarcinomas; ${ }^{\dagger} P<0.05$ vs hilar cholangiocarcinomas. (b,c) A representative case with a $K R A S$ (G12D) or IDH1 (R132C) mutation.

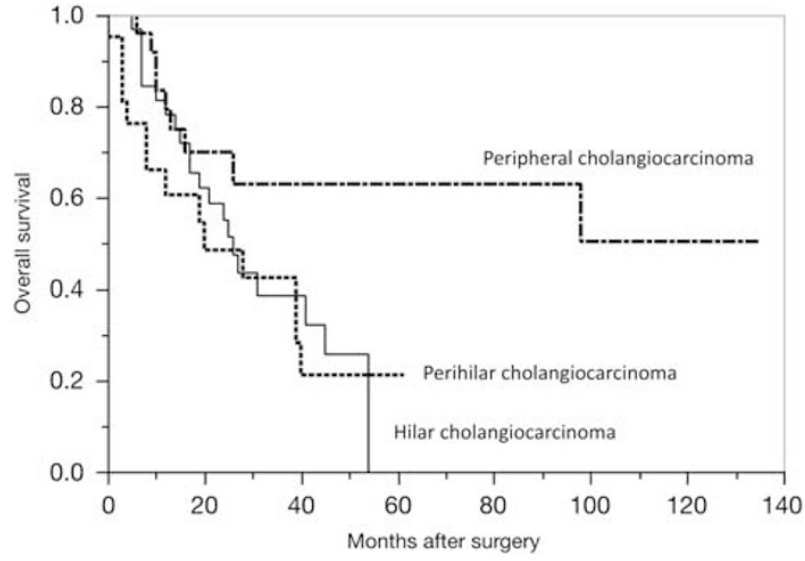

Figure 5 Survival analysis of patients with cholangiocarcinoma. Patients with peripheral cholangiocarcinoma have significantly better overall survival than those with perihilar cholangiocarcinoma. The survival curve of hilar cholangiocarcinomas appears to be very similar to that of perihilar cholangiocarcinomas.

potential prognostic factors (pT classification, lymph node metastasis, ${ }^{14}$ tumor size $(>2 \mathrm{~cm}),{ }^{15}$ and degrees of histologic differentiation $\left.{ }^{16}\right)$. Cox's proportional hazards model only identified the histologic classification as an independent prognostic factor for overall survival $(P=0.005$, HR $3.638,95 \%$ CI: 1.471-9.678; Table 2).

\section{Comparison to Marker-Based Classification Criteria}

Staining-based classification criteria of intrahepatic cholangiocarcinoma recently proposed were applied to our study cohort, to elucidate how the two separation schemes are correlated. The previously proposed classification standard ${ }^{5}$ is based on expression values of S100P, N-cadherin, NCAM, and
Table 2 Multivariate analysis of patient prognoses

\begin{tabular}{lccc}
\hline Variables & P-value & $H R$ & $95 \%$ CI \\
\hline $\begin{array}{l}\text { Histologic classification } \\
\quad \text { Perihilar vs peripheral }\end{array}$ & 0.005 & 3.638 & $1.471-9.678$ \\
$\quad \begin{array}{l}\text { p stage } \\
\text { pT }<3 \text { vs pT } \geq 3\end{array}$ & 0.100 & & \\
$\begin{array}{l}\text { Lymph node metastasis } \\
\quad \text { Present vs absent }\end{array}$ & 0.103 & \\
$\begin{array}{l}\text { Degree of differentiation } \\
\quad \text { Well-/moderately vs poorly diff }\end{array}$ & 0.232 & \\
$\begin{array}{l}\text { Tumor size } \\
\quad \leq 2 \mathrm{~cm} v \text { s }>2 \mathrm{~cm}\end{array}$ & 0.641 & \\
\hline
\end{tabular}

Abbreviations: CI, confidence interval; HR, hazard ratio.

mucin. As shown in Table 3, two schemes were moderately correlated (concordant rate, $72 \%$ ) but six cases were classified into the 'indeterminate' group on the marker-based classification. Two of them lacked the expression of either BAP1 or SMAD4. In addition, among four cases, which had discrepant results (perihilar cholangiocarcinomas in our standard, whereas small-duct cholangiocarcinomas in the marker-based scheme), one was negative for SMAD4, suggesting the perihilar type (Table 3).

\section{Discussion}

The results obtained in the present study are summarized as follows: (i) On the basis of our separation criteria, $55 \%$ of intrahepatic cholangiocarcinomas were classified as the peripheral type and $45 \%$ as the perihilar type. (ii) The two subtypes had distinct clinicopathologic features, particularly 
Table 3 Comparison between our separation scheme and markerbased classification criteria

Marker-based classification ${ }^{\mathrm{a}}$

\begin{tabular}{lccc} 
& $\begin{array}{c}\text { Small-duct } \\
\text { type }\end{array}$ & $\begin{array}{c}\text { Indeterminate } \\
\text { type }\end{array}$ & $\begin{array}{c}\text { Large-duct } \\
\text { type }\end{array}$ \\
\hline $\begin{array}{l}\text { Peripheral type }(n=25)^{\mathrm{b}} \\
\text { Perihilar type }(n=21)\end{array}$ & 23 & $4^{\mathrm{d}}$ & 0 \\
\hline
\end{tabular}

${ }^{a}$ Classification criteria available in ref. 5 .

${ }^{\mathrm{b}}$ One case could not be assessed because no tissue remained.

${ }^{\mathrm{C}}$ One case lacking BAP1 expression.

$\mathrm{d}$ One case each negative for SMAD4.

in terms of a history of chronic liver diseases, the presence of biliary intraepithelial neoplasia in adjacent bile ducts, and the immunophenotypes of tumor cells. (iii) The frequencies of mutations in $I D H 1, S M A D 4$, and BAP1 were suggested to markedly differ between the two groups. (iv) Patients with perihilar cholangiocarcinoma had worse prognoses than those with peripheral cholangiocarcinoma, with the histologic classification being identified as an independent prognostic factor. (v) Overall clinicopathologic and molecular features were similar between perihilar and hilar cholangiocarcinomas.

There is growing evidence to suggest that there are two distinct subtypes of intrahepatic cholangiocarcinoma. Previous studies have used various classification schemes, including those based on gross/ imaging features, ${ }^{3}$ histopathologic appearance, ${ }^{4}$ and immunophenotypes (eg, N-cadherin, S100P) ${ }^{5}$ (Table 4). In the present study, we defined perihilar cholangiocarcinomas as tumors histologically similar to hilar cholangiocarcinomas, according to our speculation that cancers originating from the intrahepatic large bile ducts are biologically similar to hilar cholangiocarcinomas. An interesting result was that tubular adenocarcinomas resembling reactive bile ductules might be focally present at the tumorliver interface in otherwise typical hilar cholangiocarcinomas. Although the cholangiolar morphology has been regarded as a microscopic feature suggestive of peripheral cholangiocarcinomas, the location of tubular components appears to be more important for this classification. Although reproducibility is always an issue in morphology-based classification schemes, our standard carried good agreement with the kappa value $>0.6$.

Despite the different classification criteria used in previous studies, ${ }^{3-5}$ the results obtained were similar. As shown in Table 3, three out of the four previous studies on this subject (including the present study) were from Japan, ${ }^{3,5}$ whereas the remaining was published by a Taiwanese group. ${ }^{4}$ The proportion of the perihilar type was nearly identical $(43-45 \%)$ in the three Japanese studies. Although the proportion of the perihilar type was markedly larger in the Taiwanese study (59\%), this difference may have been due to hepatolithiasis and liver flukes, which are both known risk factors for large-duct cholangiocarcinomas and are more common in Taiwan than in Japan. Collectively, these previous findings and the present results show that mutations in IDH1/2, BAP1, and FGFR2 are highly specific for peripheral cholangiocarcinomas, whereas gene abnormalities in SMAD4 are largely confined to perihilar cholangiocarcinomas.

Although it currently remains unclear which classification criteria are the most clinically useful, our standard has a couple of advantages. First, the classification is based purely on histological features, thus does not require additional staining. Second, all cases are easily classifiable with no indeterminate or intermediate cases. Another issue is that various nomenclatures have been used to describe two subgroups of intrahepatic cholangiocarcinomas: 'hilar and peripheral types', 'large-duct and smallduct types'5 and 'bile duct and cholangiolar types'. ${ }^{4}$ The standardization of nomenclatures for intrahepatic cholangiocarcinomas will require international consensus among not only pathologists but also surgeons and other clinicians.

Prior to the initiation of the present study, we suspected that KRAS may be more frequently mutated in perihilar cholangiocarcinomas than in the peripheral type. However, KRAS mutations were identified in both types at a similar frequency. In the literature, the frequencies of KRAS mutations in intrahepatic cholangiocarcinomas range between 8.6 and $25 \% .4,5,17-19$ A recent large-scale study using a whole-exome sequencing protocol detected KRAS mutations in $25 \%$ of intrahepatic cholangiocarcinomas. ${ }^{19}$ Although a histologic classification of intrahepatic cholangiocarcinomas was not performed in that study, $\sim 50 \%$ of the cases examined were supposedly of the perihilar type. By assuming that KRAS mutations only occur in the perihilar type, nearly one-half of perihilar cholangiocarcinomas must have KRAS mutations. This figure appears to be too high. Another recent study on combined hepatocellular-cholangiocarcinomas also identified KRAS mutations in $4 / 53(8 \%)$ cases. ${ }^{20}$ On the basis of biological similarities between combined liver cancers and peripheral cholangiocarcinomas, it is not unexpected that some cases of peripheral cholangiocarcinomas harbor a KRAS mutation. This driver mutation may occur more frequently in peripheral bile duct cancers than currently anticipated. Therefore, the presence of KRAS mutations does not necessarily mean that intrahepatic tumors originate from the large bile ducts.

Mutations in IDH1/2 have been increasingly identified in various tumors, ${ }^{21}$ and are known to occur in $10-22 \%$ of intrahepatic cholangiocarcinomas. ${ }^{4,5,17,22}$ The hot spots of mutations were codon 132 of IDH1 and codon 172 of IDH2. In the present study, IDH1 mutations were only observed in peripheral cholangiocarcinomas, which is consistent with previous studies. Therefore, the sequencing of IDH1 appears to be useful for the classification of intrahepatic 
Table 4 Comparison of four studies on the classification of intrahepatic cholangiocarcinomas

\begin{tabular}{|c|c|c|c|c|}
\hline & $\begin{array}{l}\text { Aishima, et al. } \\
\text { Japan }\end{array}$ & $\begin{array}{l}\text { Liau, et al. }{ }^{4} \\
\text { Taiwan }\end{array}$ & $\begin{array}{l}\text { Hayashi, et al. } \\
\text { Japan }\end{array}$ & $\begin{array}{l}\text { Present study } \\
\text { Japan }\end{array}$ \\
\hline Classification criteria & $\begin{array}{l}\text { On the basis of the gross } \\
\text { appearance. The perihilar } \\
\text { type is defined as tumors } \\
\text { involving segmental or } \\
\text { larger bile ducts, whereas } \\
\text { peripheral tumors only affect } \\
\text { smaller ducts. }\end{array}$ & $\begin{array}{l}\text { On the basis of microscopic } \\
\text { findings. The perihilar type is } \\
\text { composed of tall columnar } \\
\text { tumor cells arranged in a large } \\
\text { glandular pattern, whereas the } \\
\text { peripheral type consists of } \\
\text { cuboidal to low columnar cells } \\
\text { typically arranged in small } \\
\text { monotonous glands }\end{array}$ & $\begin{array}{l}\text { According to a scoring system, in } \\
\text { which degrees of mucin production } \\
\text { and immunohistochemical expression } \\
\text { values of S100P and N-cadherin are } \\
\text { semiquantitatively evaluated. }\end{array}$ & $\begin{array}{l}\text { On the basis of microscopic findings. } \\
\text { Perihilar tumors mainly consist of ductal } \\
\text { adenocarcinoma and minor tubular } \\
\text { components, if present, restricted to the } \\
\text { tumor-liver interface, whereas } \\
\text { peripheral cancers have tubular } \\
\text { components in the central parts of } \\
\text { tumors. }\end{array}$ \\
\hline \multicolumn{5}{|l|}{ Incidence of each type } \\
\hline Perihilar/peripheral & $44 \% / 56 \%$ & $59 \% / 41 \%$ & $43 \% / 57 \%^{a}$ & $45 \% / 55 \%$ \\
\hline \multicolumn{5}{|l|}{ Gene abnormalities } \\
\hline KRAS mutation & NA & $24 \% /<1 \%$ & $29 \% / 2 \%$ & $14 \% / 19 \%$ \\
\hline IDH1/2 mutation & NA & $5 \% / 17 \%$ & $0 \% / 40 \%$ & $0 \% / 15 \%$ \\
\hline FGFR2 translocation & NA & NA & $0 \% / 11 \%$ & NA \\
\hline Loss of BAP1 & NA & NA & NA & $5 \% / 35 \%$ \\
\hline Loss of SMAD4 & NA & NA & NA & $33 \% / 4 \%$ \\
\hline \multicolumn{5}{|l|}{ 5-year overall survival } \\
\hline Perihilar/peripheral & $36 \% / 60 \%$ & $21 \% / 37 \%$ & $20 \% / 62 \%$ & $21 \% / 63 \%$ \\
\hline
\end{tabular}

Abbreviation: NA, not available.

${ }^{a}$ Four cases that could not be classified were excluded. 
cholangiocarcinomas, with mutations indicating that the tumor is more likely to be the peripheral type. In addition, recent studies have also suggested that $I D H 1 / 2$ mutations are associated with better overall survival in patients with intrahepatic cholangiocarcinoma. ${ }^{23,24}$ In our cohort, although tumor recurrence was observed in a single case, all patients with IDH1-mutated peripheral cholangiocarcinoma were alive. IDH1/2 are also interesting from a therapeutic aspect because specific inhibitors for IDH1 (AG-120) and IDH2 (AG-221) are currently being investigated in phase I (NCT02073994) and phase I/II (NCT02273739) clinical trials, respectively, for cholangiocarcinomas. ${ }^{25}$ This approach may be promising because the inhibition of mutant IDH1 was previously reported to decrease the tumor proliferation of glioma cells in vitro. ${ }^{26}$

The comparison between our separation scheme and marker-based criteria $^{5}$ identified moderate correlation (Table 3). We found a significant difference in S100P expression between the present study and the original data. S100P was positive in $56 \%$ of cases in the original study, whereas it was expressed in only $13 \%$ in our study. This discrepancy may be due to the difference of antibodies used. Of ten cases with discrepant or indeterminate results, three cases lacked the expression of either SMAD4 or BAP1, suggesting that immunostaining for these molecules highly specific for either subtype may also be useful in marker-based classification. This will be particularly helpful in biopsy cases, for which morphology-based classification is not always possible.

There is a limitation to the present study. Similar to the histologic classification criteria used in other studies, our separation scheme requires surgically resected specimens. Therefore, the classification of tumors cannot be made in non-surgical cases. Some radiological studies that previously attempted to classify intrahepatic cholangiocarcinomas based on imaging features revealed that several radiological findings correlated with patient prognoses. ${ }^{27-29} \mathrm{~A}$ radiology-pathology correlation study is currently underway to identify imaging features that correlate with our histologic classification of intrahepatic cholangiocarcinomas.

In conclusion, this study revealed that there are two distinct subtypes of intrahepatic cholangiocarcinomas. The perihilar type appears to share clinicopathologic and molecular features with hilar cholangiocarcinomas. This histologic classification will be clinically relevant given its independent prognostic value. Different clinical management strategies may be considered in the future based on the histologic classification.

\section{Disclosure/conflict of interest}

The authors declare no conflict of interest.

\section{References}

1 Lee CH, Hsieh SY, Chang CJ, et al. Comparison of clinical characteristics of combined hepatocellularcholangiocarcinoma and other primary liver cancers. J Gastroenterol Hepatol 2013;28:122-127.

2 Yoon YI, Hwang S, Lee YJ, et al. Postresection outcomes of combined hepatocellular carcinoma-cholangiocarcinoma, hepatocellular carcinoma and intrahepatic cholangiocarcinoma. J Gastrointest Surg 2016;20:411-420.

3 Aishima S, Kuroda Y, Nishihara Y, et al. Proposal of progression model for intrahepatic cholangiocarcinoma: clinicopathologic differences between hilar type and peripheral type. Am J Surg Pathol 2007;31: 1059-1067.

4 Liau JY, Tsai JH, Yuan RH, et al. Morphological subclassification of intrahepatic cholangiocarcinoma: etiological, clinicopathological, and molecular features. Mod Pathol 2014;27:1163-1173.

5 Hayashi A, Misumi K, Shibahara J, et al. Distinct clinicopathologic and genetic features of 2 histologic subtypes of intrahepatic cholangiocarcinoma. Am J Surg Pathol 2016;40:1021-1030.

6 Murakami Y, Uemura K, Sudo T, et al. Intrahepatic cholangiocarcinoma: clinicopathological differences between peripheral type and hilar type. J Gastrointest Surg 2012;16:540-548.

7 Aishima S, Fujita N, Mano Y, et al. Different roles of S100P overexpression in intrahepatic cholangiocarcinoma: carcinogenesis of perihilar type and aggressive behavior of peripheral type. Am J Surg Pathol 2011;35: 590-598.

8 Tsai JH, Huang WC, Kuo KT, et al. S100P immunostaining identifies a subset of peripheral-type intrahepatic cholangiocarcinomas with morphological and molecular features similar to those of perihilar and extrahepatic cholangiocarcinomas. Histopathology 2012;61:1106-1116.

9 Zen Y, Britton D, Mitra V, et al. Tubulin $\beta$-III: a novel immunohistochemical marker for intrahepatic peripheral cholangiocarcinoma. Histopathology 2014;65: 784-792.

10 Fujikura K, Fukumoto T, Ajiki T, et al. Comparative clinicopathological study of biliary intraductal papillary neoplasms and papillary cholangiocarcinomas. Histopathology 2016;69:950-961.

11 Vauthey JN, Pawlik TM, Abdalla EK et al. Intrahepatic bile ducts. In: Edge SB, Byrd DR, Compton CC (eds). AJCC Cancer Staging Manual, 7th edn. Springer: Chicago, IL, USA, 2010, pp 201-205.

12 Iacobuzio-Donahue CA, Wilentz RE, Argani $\mathrm{P}$, et al. Dpc4 protein in mucinous cystic neoplasms of the pancreas: frequent loss of expression in invasive carcinomas suggests a role in genetic progression. Am J Surg Pathol 2000;24:1544-1548.

13 Andrici J, Goeppert B, Sioson L, et al. Loss of BAP1 expression occurs frequently in intrahepatic cholangiocarcinoma. Medicine 2016;95:e2491.

14 Shimada K, Sano T, Sakamoto Y, et al. Surgical outcomes of the mass-forming plus periductal infiltrating types of intrahepatic cholangiocarcinoma: a comparative study with the typical mass-forming type of intrahepatic cholangiocarcinoma. World J Surg 2007;31:2016-2022.

15 Sakamoto Y, Kokudo N, Matsuyama Y, et al. Proposal of a new staging system for intrahepatic 
cholangiocarcinoma: Analysis of surgical patients from a nationwide survey of the Liver Cancer Study Group of Japan. Cancer 2016;122:61-70.

16 Shirabe K, Mano Y, Taketomi A, et al. Clinicopathological prognostic factors after hepatectomy for patients with mass-forming type intrahepatic cholangiocarcinoma: relevance of the lymphatic invasion index. Ann Surg Oncol 2010;17:1816-1822.

17 Zhu AX, Borger DR, Kim Y, et al. Genomic profiling of intrahepatic cholangiocarcinoma: refining prognosis and identifying therapeutic targets. Ann Surg Oncol 2014;21:3827-3834.

18 Chen TC, Jan YY, Yeh TS. K-ras mutation is strongly associated with perineural invasion and represents an independent prognostic factor of intrahepatic cholangiocarcinoma after hepatectomy. Ann Surg Oncol 2012;19:S675-S681.

19 Nakamura H, Arai Y, Totoki Y, et al. Genomic spectra of biliary tract cancer. Nat Genet 2015;47:1003-1010.

20 Sasaki M, Sato Y, Nakanuma Y. Mutational landscape of combined hepatocellular-cholangiocarcinoma and its clinicopathological significance. Histopathology 2017;70:423-434.

21 Saha SK, Parachoniak CA, Bardeesy N. IDH mutations in liver cell plasticity and biliary cancer. Cell Cycle 2014;13:3176-3182.

22 Borger DR, Tanabe KK, Fan KC, et al. Frequent mutation of isocitrate dehydrogenase (IDH)1 and IDH2 in cholangiocarcinoma identified through broad-based tumor genotyping. Oncologist 2012;17:72-79.
23 Wang P, Dong Q, Zhang C, et al. Mutations in isocitrate dehydrogenase 1 and 2 occur frequently in intrahepatic cholangiocarcinomas and share hypermethylation targets with glioblastomas. Oncogene 2013;32: 3091-3100.

24 Kipp BR, Voss JS, Kerr SE, et al. Isocitrate dehydrogenase 1 and 2 mutations in cholangiocarcinoma. Hum Pathol 2012;43:1552-1558.

25 Rohle D, Popovici-Muller J, Palaskas N, et al. An inhibitor of mutant IDH1 delays growth and promotes differentiation of glioma cells. Science 2013;340: 626-630.

26 Moeini A, Sia D, Bardeesy N, et al. Molecular pathogenesis and targeted therapies for intrahepatic cholangiocarcinoma. Clin Cancer Res 2016;22:291-300.

27 Ariizumi S, Kotera Y, Takahashi Y, et al. Mass-forming intrahepatic cholangiocarcinoma with marked enhancement on arterial-phase computed tomography reflects favorable surgical outcomes. J Surg Oncol 2011;104:130-139.

28 Türkoğlu MA, Yamamoto Y, Sugiura T, et al. The favorable prognosis after operative resection of hypervascular intrahepatic cholangiocarcinoma: A clinicopathologic and immunohistochemical study. Surgery 2016;160:683-690.

29 Baheti AD, Tirumani SH, Shinagare AB, et al. Correlation of CT patterns of primary intrahepatic cholangiocarcinoma at the time of presentation with the metastatic spread and clinical outcomes: retrospective study of 92 patients. Abdom Imaging 2014;39:1193-1201. 